\title{
Recognition memory: One-component strength functions in the "short-term to long-term transition region"
}

\author{
ADAM V. REED \\ University of Oregon, Eugene, Oregon 97409
}

\begin{abstract}
Experimental manipulation of the upper bound on the retention interval $(30 \mathrm{sec}$, with average duration of $11 \mathrm{sec}$, vs. $2 \mathrm{~min}$, with average duration of $18 \mathrm{sec}$ ) failed to produce evidence for independent adjustment of initial long-term and short-term storage strengths. Very accurate strength functions of retention time were obtained; these were fitted equally well by a two-component equation and a one-component equation derived from a theory postulating sequential employment of an active attentional buffer and a one-trace passive storage system. The latter theory appears to be capable of accounting for both post-attentional and two-phase experimental strength data, using fewer free parameters than strength theories which postulate the simultaneous existence of short-term and long-term traces. Other arguments for two traces are also discussed in relation to the postulate of a single post-attentional trace.
\end{abstract}

The validity of most arguments which have been advanced to support the notion of parallel short-term and long-term storage mechanisms in human memory has been put into question by Wickelgren (1973), who has shown that evidence usually cited in support of all but three of these arguments is equally consistent with a one-trace hypothesis. Moreover, the three arguments which Wickelgren (1973) accepted as valid are also subject to plausible objections.

Consider first the argument from long-term acquisition amnesia [Milner's (1967) H.M. and similar cases] In this syndrome, the patient is unable to recall newly learned materials after a time exceeding the presumed life of the "short-term trace" has elapsed. However, if we assume that a certain minimum memory strength is necessary for recall, then recall data for H.M. and similar cases may be explained in a manner consistent with a one-trace hypothesis. It is conceivable that H.M.'s memory strength decays at the same rate as in normals (the decay rate characteristic of the unitary mechanism), but starts out at a lower level. In the latter case, the ratio of H.M.'s memory strength to that of normals should not change over time. Wickelgren (1974b) administered to H.M. a continuous word recognition task also administered to normal subjects (Wickelgren, 1972). There is no consistent variation in the ratio of memory strength for H.M. and normal subjects as a function of time (Spearman rho $=.396$, n.s.).

This research was supported by a National Science Foundation Graduate Fellowship to the author, and by Grants MH17958-03 and NIE3-0097 to A. Wickelgren. The manuscript was prepared for publication while the author was at Rockefeller University, with support from NIH Training Grant GM01789. The author wishes to express his gratitude to Professor Wickelgren for advice, supervision, and support. Requests for reprints should be sent to Adam V. Reed, Mathematical Psychology Laboratory, Rockefeller University, New York, New York 10021.
A second argument accepted by Wickelgren (1973) is based on a differential effect of similarity on storage interference in studies of short-term memory and longterm memory. While storage interference in short-term memory appears to be independent of "fine-grain" similarity of the interpolated material to the original material, in long-term memory such similarity appears to enhance interference. This finding may be explained without recourse to more than one storage mechanism if, under conditions of long-term memory studies, memory is mediated by a larger number of fine-grain associations than in short-term studies. This difference, in turn, may be the result of differences in materials and study conditions used in the two types of memory experiments. In most experiments memory strength (or the corresponding error rate) is not measurable if $\mathrm{d}^{\prime}$ is greater than about 5.0 or less than about .5; and experiments are designed so that memory strength will be in that range when measured. Since usually there is more loss in long-term memory studies, such studies are designed to permit a greater degree of initial acquisition, and this may be achieved by permitting the formation of more associations. Thus, the phenomenon on which this argument is based may be an experimental artifact.

The final argument of Wickelgren (1973) is based on the form of retention functions. According to Wickelgren's strength theory, the total strength of an item in storage is the sum of the strengths of all the separate stored traces of that item. Hence, the existence of two storage systems following different dynamic equations would lead to a global strength retention function consisting of the sum of two components, the form of each component being identical to the dynamic strength function which would result from the operation of the corresponding storage system alone. Strength functions obtained by Wickelgren in the short-term to 
long-term transition region can indeed be fitted by two-component strength functions, with one component corresponding to an equation capable of describing long-term data from many experiments (Wickelgren, $1972,1974 a)$ and the other decaying exponentially as postulated for the short-term trace on the basis of twophase data (Wickelgren, 1970, 1974a), where the term "two-phase" refers to experiments without a separate retention phase between acquisition and retrieval phases. However, existing data do not exclude the possibility that a one-component equation might be sufficient, if an appropriate one-component equation (one consistent with the known form of short- and long-term strength functions) were found. In sum, then, none of the arguments for two distinct memory mechanisms is entirely satisfactory.

The aim of the present experiment was to investigate a conjecture which offered some hope of producing a more substantial argument for a two-trace theory. In a study which eliminated selective perception and selective rehearsal as explanations of the effect of setto-learn, Brown (1954) concluded that set had an effect on the initial storage strength of new information. In a more recent study which provided subjects with advance cues about the length of the coming retention interval, Hinrichs and Grunke (1975) found an improvement of retention in the cued condition for intervals of up to $12 \mathrm{sec}$. Hinrichs and Grunke used a paired associate paradigm without filling the retention interval with a rehearsal-preventing task, and ascribed their results to selective rehearsal. Their design, however, did not exclude differential short-term and long-term acquisition levels as an alternative explanation. In view of the obvious functional advantages of optimizing short- and long-term acquisition strengths separately (e.g., to reduce proactive interference), it seemed reasonable to investigate the possibility that the two initial strengths could be independently adjusted by the subject, as a result of set, to retain particular items for varying amounts of time. A demonstration that long- and short-term acquisition levels could vary independently of each other would have provided conclusive evidence of two separate storage mechanisms. A failure of this hypothesis would be consistent with one-trace as well as two-trace theories and would motivate a re-investigation of the argument for two traces from two-component retention functions. Accordingly, the experiment was designed to yield low-variance empirical retention functions for a quantitative comparison of two-component and onecomponent theories.

The hypothesis, that the acquisition levels of shortterm and long-term traces are subject to independent control by set-to-retain, was tested by manipulating the upper bound on the retention interval and using memory strength measurements at various retention times below this upper bound to determine the strength ratios of the long- and short-term components in each condition. Two conditions were used. In the short upper bound condition, no retention interval was longer than $30.7 \mathrm{sec}$; the mean duration of the retention interval was $11.0 \mathrm{sec}$ [which, according to the theory of Wickelgren (1973), should be within the lifetime of the short-term trace]. In the long upper bound condition, retention intervals ranged up to $2 \mathrm{~min}$, with a mean duration of $18.1 \mathrm{sec}$.

\section{METHOD}

\section{Procedures and Materials}

Each trial began with a verbal warning, "ready," spoken by the experimenter. The warning was followed by the presentation of a trigram of consonants on an electroluminescent panel. The trigram was displayed for $1 \mathrm{sec}$, and was read aloud by the subjects. It was then replaced on the display panel by a randomly generated three-digit number, between 488 and 999 , which was also read aloud by the subjects, who immediately began to count backward by threes at a self-paced rate of one count/sec or higher. After the number had been displayed for $1 \mathrm{sec}$, the panel was blanked, and remained blank for the remainder of the retention interval, which varied from 3 to $120 \mathrm{sec}$. The retention interval was terminated by the display of the probe consonant on a separate panel. The probability that the probe consonant had been a member of the trigram presented at the beginning of the trial was .5. Subjects were instructed to decide as rapidly as they could whether the probe consonant had been a member of the trigram, and to rate their confidence on a scale from 1 (most certain) to 4 (least). They were instructed to use the four confidence categories equally often. The subjects recorded their answers by pressing the appropriate lever (marked "Y1," "N3," etc.) on a manual computer-card punch. The retention interval was measured from offset of trigram to onset of probe. Trigram members were chosen without replacement from among the 16 consonants $B$, C, D, F, G, H, K, L, M, N, P, R, S, T, V, and Z.

The subjects were instructed to refrain from rehearsing or thinking about consonants while counting. They were instructed to restart at 999 if they lost track or ran out of numbers while counting. The subjects counted in a low whisper into hand-held microphones, which were monitored on each trial, and the subjects did not know when they were being monitored. Subjects who counted at a rate slower than one three-digit number every $.75 \mathrm{sec}$ were asked to count faster. After the conclusion of the initial training sequence, prompting to count faster was not required more than three times with any subject. No record was kept of monitored counting rates, which appeared to be homogeneous across the two upper bound conditions. The subjects did not know in advance how long each particular trial was going to last, except for the upper limit.

\section{Design}

Each session of the experiment consisted of 96 trials and lasted approximately $1 \mathrm{~h}$. Each subject participated in 50 sessions, held at the same time of day on the 5 weekdays of 10 consecutive weeks. Make-up sessions for subjects who missed a regular session were held on weekends. The subjects were divided into two groups of four subjects each. Each group participated in 27 sessions in one upper bound condition, the first 7 of which were practice sessions, followed by 23 sessions in the other upper bound condition, the first 3 of which were practice. The order of upper bound conditions was reversed between groups. The design of each short $(30-\mathrm{sec})$ upper bound session was derived, by transformation, from the design of the long (2-min) upper bound session used with the other group of subjects on the same day. Trials with retention intervals $30.7 \mathrm{sec}$ or less were unchanged by the transformation. Every trial longer than this limit was replaced by a trial with a retention interval of $8 \mathrm{sec}$, using the same trigram, number, and 
probe. The extra time was filled with backward counting. For example, a trial with retention interval of $91 \mathrm{sec}$ would be replaced by one with retention interval of $8 \mathrm{sec}$, which would be preceded by 83 additional sec of backward counting. Thus, the sequence and density of stimuli presented and the total amount of backward counting were exactly the same in the two conditions.

Three balanced 20-session pseudorandom blocks of trials were generated for the experiment. The first block was used for the working sessions in the first upper bound condition for each group (Sessions 8 through 27), the second for working sessions in the second upper bound condition (Sessions 31 through 50 ), and the third was reserved for equipment tests, practice sessions, etc.

\section{Subjects}

The eight subjects were University of Oregon undergraduates, and were paid for their services. The four subjects in each group were usually tested together. They were separated from each other by wooden panels, and wore "Sound Barrier" industrial hearing protectors.

\section{RESULTS AND DISCUSSION}

\section{Data Reduction}

Throughout this article, the signal-to-noise ratio $\mathrm{d}^{\prime}$ [corresponding to the square root of $(\mathrm{S} / \mathrm{N})_{0}$ ] will be used as the empirical measure of memory strength. The $d_{a}$, an estimate of $d^{\prime}$ developed by W. A. Wickelgren (1972), was employed throughout this experiment. In the $d_{a}$ procedure, each set of ROC points is first fitted with a linear ROC in the probability-probability plane. A family of ROC lines with log slope proportional to $d_{s}$ is then generated, the constant of proportionality being determined by regression of the log slope of the initial ROC lines on their $d_{s}$. Each set of ROC points is then fitted with the best-fitting line belonging to that family. The $d_{a}$ is the noise-axis intercept, in standard deviates, of this last line.

Theoretical curves were compared to empirical curves obtained by logarithmic (geometric) averaging of $d_{a}$ 's across subjects. Logarithmic rather than linear averaging was used because retention functions are usually of the form

$$
d_{i}=a_{i} e^{b} b_{j} f(t)
$$

where $f(t)$ are particular functions of retention time. If the expected retention curve for the ith subject is of that form, the logarithmic (geometric) average of the $n$ individual curves will be of the form

$$
D=A e^{B} f(t)
$$

where

$$
\begin{aligned}
& D \triangleq \Pi_{i=1}^{n} d_{i}^{1 / n} \\
& A \triangleq n_{i=1}^{n} a_{i}^{1 / n}
\end{aligned}
$$

$$
B \triangleq 1 / n \sum_{i=1}^{n} b_{i}
$$

and $f(t)$ is the same as for individual curves. Thus, if logarithmic averaging is used, the expected form of the averaged empirical curves should correspond exactly to one-component theoretical curves, such as those introduced later in the discussion. For two-component theoretical curves, the agreement should be exact in the limit, as one component is much greater than the other, and approximate elsewhere. The equations were fitted to logarithmically averaged data with a hillclimbing program; and $\mathrm{r}^{2}$, the percentage of variance accounted for, was adjusted for the number of degrees of freedom remaining after subtraction of $k$ free parameters according to the formula

$$
r^{2}=1-\frac{\left[\sum_{i=1}^{n}\left(x_{i}-\hat{x}_{i}\right)^{2}\right] /(n-k)}{\left[\sum_{i=1}^{n}\left(x_{i}-\bar{x}\right)^{2}\right] /(n-1)}
$$

where $\hat{\mathrm{x}}_{\mathrm{i}}$ is the fitted value corresponding to $\mathrm{x}_{\mathrm{i}}$.

\section{Effects of Long and Short Upper Bounds}

Subjective difficulty. Although verbal comments from subjects concerning the relative difficulty of the high and low upper bound tasks were not solicited, all subjects in the group that was switched from the long upper bound to the short spontaneously reported that the latter was easier. A typical comment was "After the old stuff, this is a breeze." Opposite spontaneous comments were received from the group that was tested on the short upper bound task first and then switched to long upper bound. Two of the four subjects in the latter group refused to continue their participation in the experiment, and returned only when a $\$ 20$ bonus was offered to all subjects for completion of the remaining work.

Strength retention curves. The responses made in the 20 working sessions of each upper bound condition were used to compute $d_{a}$ strength estimates for each retention interval up to $30.7 \mathrm{sec}$ for each subject. Reliable estimates of $d_{a}$ could not be computed for longer retention intervals because of the small number of long trials. Logarithmically averaged retention curves for the two upper bound conditions are compared in Figure 1a. If the short upper bound had resulted in a greater proportion of the total strength being assigned to the short-term trace, the ratio of the short upper bound to the long upper bound retention strength should decrease with retention time. In fact, however, no systematic difference between the two curves could be discerned. This result confirms differential rehearsal as the mechanism by which retention interval information affects short-term retention in experiments such as Hinrichs and Grunke (1975). Even if two traces were 


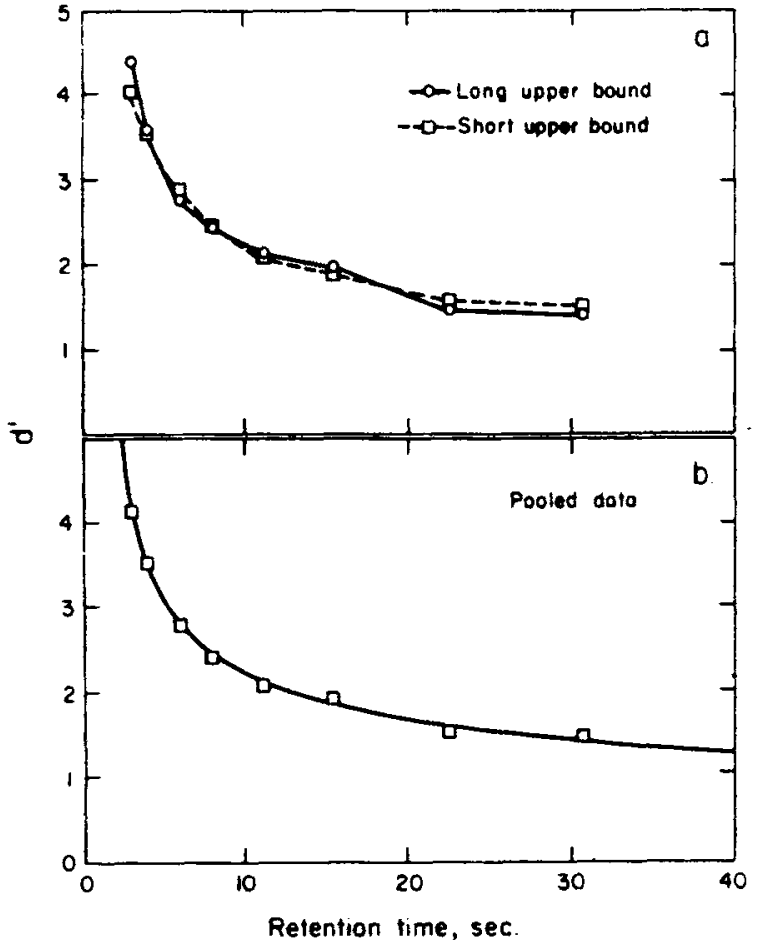

Figure 1. Recognition memory strength functions obtained in the present experiment. Top panel: A comparison of retention functions obtained in conditions with $30-\sec$ and $120-\sec$ upper bounds on the retention interval. Bottom panel: Pooled data strength function, with curve of Equation 5.

present, their strengths do not appear to be independently variable under the conditions of the present experiment.

\section{Pooled Data and Differential Evaluation of}

Two-Component and One-Component Theories

Pooled data. On finding no significant difference between the two upper bound conditions, the raw data from the two conditions were pooled, the $d_{a}$ 's recomputed, and the results used for differential evaluation of various strength theories.

Comparison with two-component strength-resistance theory. The strength-resistance theory of Wickelgren (1974a) postulates the existence of two traces, existing simultaneously and formed at the instant of acquisition. The strength of the short-term trace is assumed to decay with time at a rate proportional only to its current value, i.e., exponentially. The strength of the long-term trace is assumed to decay at a rate proportional to its current strength and inversely proportional to a resistance parameter, which grows with time. If one assumes that this resistance parameter is proportional to time since acquisition, the total strength of the two traces should equal

$$
d^{\prime}=\alpha e^{-\beta t}+\lambda t^{-\psi}
$$

where $\alpha$ is the initial strength of the short-term trace, $\lambda$ is the strength of the long-term trace at $t=1$, and $\beta$ and $\psi$ are the decay parameters of the two traces. This equation was fitted to data and the resulting parameters, shown at the top of Table 1 , are within the range of values reported by Wickelgren. With an $r^{2}$ of .995 , the two-component theory is clearly adequate to describe the data.

Although the two-trace theory does describe the data, it is in a sense suboptimal: Since the acquisition parameters postulated by the theory are not subject to independent cognitive control, their ratio becomes a superfluous free parameter. Wickelgren (1974b) has already reported an original single-trace theory inspired in part by preliminary results from the present investigation. Another theory, combining a single postattentional dynamic trace with a traditional attentional buffer, is presented below.

A one-component theory of postattentional storage. Wickelgren (1974a), like many other memory theorists (see Kintsch, 1971, for a review), assumes that the decay of the "long-term trace" begins at the moment an item is first acquired by the subject. By rejecting this assumption, we may account for strength curves obtained in the short-term to long-term transition region with a one-component equation having the dynamics previously postulated for the long-term trace. The theory below assumes that a newly acquired item is held in an active limited-capacity attentional buffer until displaced by subsequent entries. The decay of strength and the growth of resistance do not begin until after an item is transferred from the attentional buffer, into storage, at time $\theta$. The resulting strength equation is

$$
d^{\prime}(t)=\lambda(t-\theta)^{-\psi}
$$

Note that for $t>\theta$ the predictions of this onecomponent theory and of Wickelgren's strengthresistance theory converge, so that all long-term memory results accounted for by Wickelgren $(1972,1974 a)$ are also consistent with the present theory.

Table 1

Parameter Fitting

\begin{tabular}{lccccc}
\hline Parameters of Equation 4: & $\alpha$ & $\beta$ & $\lambda$ & $\psi$ & $\mathrm{r}^{2}$ \\
\hline Present experiment: CCC & 1.22 & .0047 & 7.54 & .858 & .995 \\
\hline Parameters of Equation 5: & $\theta$ & $\lambda$ & $\psi$ & $\mathrm{r}^{2}$ \\
\hline Present experiment:CCC & 1.45 & 4.86 & .362 & .995 \\
First C in CCC & 1.22 & 4.78 & .348 & .994 \\
Second C in CCC & 1.88 & 4.37 & .333 & .993 \\
Third C in CCC & 1.09 & 4.87 & .380 & .988 \\
Wickelgren and Berian, 1971: & & & & \\
One consonant & 4.73 & 6.92 & .338 & .968 \\
Six consonants & 1.13 & 2.93 & .383 & .938 \\
Wickelgren, 1972: & & & & \\
Continuous words & 2.54 & 5.11 & .132 & .868 \\
Continuous CCCDDD & 3.33 & 2.61 & .201 & .970 \\
\hline Parameters of Equation 6: & $\mathrm{n}$ & $\mathrm{t}_{\mathrm{o}}$ & $\lambda$ & $\psi$ & $\mathrm{r}^{2}$ \\
\hline
\end{tabular}

Wickelgren, 1970:

$\begin{array}{llllll}\text { Two phase, } 15 \text { consonants } & 4.57 & .029 & .215 & .832 & .971\end{array}$ 
Comparison of data with the one-component theory. As in the case of the two-component theory, the equation for the one-component theory was fitted to the pooled data. The curve of the one-component equation is compared with data in Figure $\mathbf{l b}$. The parameters are again shown in Table 1 . The value of $\theta$ obtained by fitting the one-component equation to data is $1.45 \mathrm{sec}$. This appears plausible, since the first second of the retention interval is used to read the starting number and the contents of the activation buffer might begin to be displaced within $1 / 2 \mathrm{sec}$ after the start of backward counting. The $r^{2}$ is again .995 . To check the validity of Equation 5 under different experimental conditions, it was also fitted to the four sets of retention data from Wickelgren and Berian (1971) and Wickelgren (1972). These experiments used auditory as well as visual presentation, continuous as well as three-phase (presentation-retention-retrieval) designs, and a variety of materials (consonants, words, and numbers). The onecomponent theory of Equation 5 gave an adequate fit to all the data from these experiments, with parameters not far from those used to fit the empirical curves from the present one. The results are included in Table 1.

Two-phase data. Wickelgren (1974a) has pointed out that strength curves from experiments in which the test phase immediately follows the learning phase (the socalled "two-phase" experiments) cannot be fitted by equations describing postattentional curves. In twophase experiments, memory strength appears to decay in the manner of a simple exponential with elapsed time, the time constant depending on the rate at which additional items are presented during the interval between the given item and the probe. In view of the fact that two-phase experiments do not use a separate rehearsal-preventing task, the above finding may be made consistent with the present theory by assuming that some of the memory strength found in those experiments is due to attentional activation rather than to postattentional memory. In order to describe the results of two-phase experiments in terms of the sequential one-component-at-a-time theory being proposed here, additional assumptions must be made. In particular, the process determining the time at which an item is transferred from attentional to postattentional memory must be specified in more detail.

It will be assumed, following Atkinson and Shiffrin (1968), that items are displaced out of the activation buffer at the moment of arrival of new items, the capacity of attentional memory being limited to $n$ items at a time. For any one subject the capacity parameter $\mathrm{n}$ will be an integer, although it may assume noninteger values when data are averaged across subjects. Depending on the strategy chosen by the subjects, the displaced item might be selected either at random or on a first in, first out basis. In analyzing strength curves for the first, second, and third position within CCC in the present study, it was found that $\theta$, the time of transfer from attentional to postattentional memory, did not vary systematically with input order (see Table 1). Therefore, it will be assumed that the process of selecting items for transfer is essentially random. Although the actual probability of transfer might be a function of time already spent in attentional memory, the following discussion will assume, for simplicity, that the probability of any given item being transferred out is $1 / \mathrm{n}$.

It will be further assumed that the strength of an item in attentional memory stays constant until it is transferred out, that the growth of resistance begins at the moment of transfer, and that items are transferred at strength and begin to decay at time $t_{0}$ such that $\lambda t_{0}-\psi$ equals the starting strength. In two-phase experiments, the average expected strength is

$$
\mathrm{d}^{\prime}=\lambda\left[\left(1-\frac{1}{\mathrm{n}}\right)^{\mathrm{m}} \mathrm{t}_{\mathrm{o}}{ }^{-\psi}+\sum_{\mathrm{i}=1}^{\mathrm{m}}\left(1-\frac{1}{\mathrm{n}}\right)^{\mathrm{m}-\mathrm{i}}\left(\frac{1}{\mathrm{n}}\right)(\mathrm{it})^{-\psi}\right]
$$

where $\lambda$ and $\psi$ are defined as in Equation 5, $\mathrm{n}$ is the capacity of attentional memory, $\mathrm{m}$ is the number of items intervening between the given item and probe, $t$ is the length of the time segment between successive items, and $i$ is the number of such time segments (if any) since the transfer of the given item into postattentional memory. The first item in Equation 6 is the "starting strength" times the likelihood that a given item is still in the attentional buffer; subsequent terms come from transfers $i$ items ago.

In Figure 2, Equation 6 is fitted to the two-phase 15-consonant list data of Wickelgren (1970), averaged logarithmically across all subjects and both distractor conditions (compare Figure 1 of Wickelgren, 1974a). The parameters are shown in Table 1. The two-phase

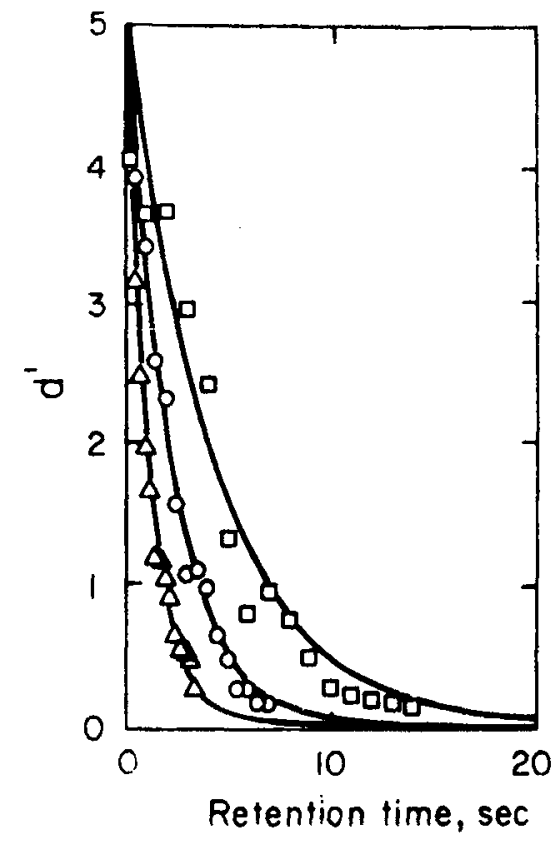

Figure 2. Comparison of two-phase retention functions obtained by Wickelgren (1970) with the curves of Equation 6 . Data for $1 / \mathrm{sec}$ (squares), $2 / \mathrm{sec}$ (circles), and $4 / \mathrm{sec}$ (triangles) presentation rates. Compare with Figure 1 of Wickelgren (1974a). 
data are satisfactorily fitted using only four parameters (in this case, $\lambda, \psi, t_{0}$, and $n$ ). Those parameters are independent of presentation rate, as long as the latter is slow enough to permit acquisition of every item and fast enough to hold the subject's attention. The three experimental curves, corresponding to presentation rates of one, two, and four items/sec, are fitted with one set of parameters. In summary, a single postattentional trace appears capable of explaining two-phase as well as threephase and continuous recognition data. Thus, there appears to be no reason to assume, in the context of strength theories of human memory, the existence of more than one postattentional storage mechanism.

\section{REFERENCES}

Atrinson, R. C., \& Shiffrin, R. M. Human memory: A proposed system and its control processes. In K. W. Spence \& J. T. Spence (Eds.). The psychology of learning and motivation: Research and theory (Vol. II). New York: Academic Press, 1968.

Brown, I. The nature of set-to-learn and of intra-material interference in immediate memory. Quarterly Joumal of Experimental Psychology, 1954, 6, 141-148.

Hinrichs, James V., \& Grunke, Mary E. Control processes in short-term memory: Use of retention interval information. Journal of Experimental Psychology, 1975, 104, 229-237.

Kintsch, W. Learning, memory, and conceptual processes. New York: Wiley, 1971.

Milner, B. Amnesia following operation on the temporal lobes. In O. L. Zangwill \& C. W. M. Whitty (Eds.), Amnesia. London: Butterworth, 1967

Wickelgren, W. A. Time, interference and rate of presentation in short-term recognition memory for items. Joumal of Mathematical Psychology. 1970, 7, 219-235.

Wickelgren. W. A. Trace resistance and the decay of longterm memory. Journal of Mathematical Psychology. 1972, 9, 418-455.

Wickelgren, W. A. The long and the short of memory. Psychological Bulletin, 1973, 80, 425-438.

WICKELGREN, W. A. Strength-resistance theory of the dynamics of memory storage. In D. H. Krantz, R. C. Atkinson, R. D. Luce, \& P. Suppes (Eds.), Contemporary developments in mathematical psychology. New York: Freeman, 1974. (a)

WiCKELGREN. W. A. Single-trace fragility theory of memory dynamics. Memory \& Cognition, 1974, 2, 775-780. (b)

Wickelgren, W. A.. \& Berian, K. M. Dual trace theory and the consolidation of long-term memory. Journal of Mathematical Psychology, 1971. 8. 404-417.

(Received for publication August 11, 1975; revision accepted November 24, 1975.) 